\title{
MINIMALITY OF PLANES IN NORMED SPACES
}

\author{
DMITRI BURAGO AND SERGEI IVANOV
}

\begin{abstract}
We prove that a region in a two-dimensional affine subspace of a normed space $V$ has the least 2-dimensional Hausdorff measure among all compact surfaces with the same boundary. Furthermore, the 2-dimensional Hausdorff area density admits a convex extension to $\Lambda^{2} V$. The proof is based on a (probably) new inequality for the Euclidean area of a convex centrallysymmetric polygon.
\end{abstract}

\section{INTRODUCTION}

The main purpose of this paper is to prove the following seemingly elementary fact. Consider a bounded region in a two-dimensional affine plane in a normed vector space. Then the region has the least possible two-dimensional Hausdorff measure among all compact two-dimensional surfaces with the same boundary. Even though the problem sounds almost silly, it stood open for over 50 years and still remains open in dimensions greater than 2.

We prove our result by showing the convexity of the density function for the Busemann-Hausdorff surface area in normed spaces. This contrasts with a result of Busemann, Ewald and Shephard [7] who demonstrated that the density of the Holmes-Thompson (symplectic) surface area may fail to be convex.

We first embed the problem in a more general set-up, borrowing some preliminaries from [3]. Here we consider various notions of $k$-dimensional surface areas, although the rest of the paper is devoted to the case $k=2$ and the BusemannHausdorff definition of area in a normed space.

A common way to introduce a (translation-invariant) $k$-dimensional surface area in $\mathbb{R}^{n}$ is to define its density in every $k$-dimensional linear subspace. Namely for a continuous function $A: G(n, k) \rightarrow \mathbb{R}_{+}$, where $G(n, k)=G_{k}\left(\mathbb{R}^{n}\right)$ is the Grassmannian manifold of $k$-dimensional linear subspaces of $\mathbb{R}^{n}$, one defines the associated surface area functional $\mathrm{Area}_{A}$ by the obvious formula

$$
\operatorname{Area}_{A}(S)=\int_{S} A\left(T_{x} S\right) d m(x)
$$

where $S$ is a smooth (more generally, Lipschitz) surface, $m$ is the $k$-dimensional Euclidean surface area, and the tangent spaces $T_{x} S$ are regarded as points in $G(n, k)$.

If $\mathbb{R}^{n}$ is equipped with a norm $\|\cdot\|$, then every subspace $P \in G(n, k)$ becomes a $k$-dimensional normed space. In the Busemann-Hausdorff definition of area, the density $A(P)$ is defined so that the area of the norm's unit ball (in $P$ ) equals the standard constant $\varepsilon_{k}$ depending only on $k$, namely $\varepsilon_{k}$ is the Euclidean volume of

2010 Mathematics Subject Classification. 52A21, 52A38, 52A40, 53C60.

Key words and phrases. Busemann-Hausdorff surface area, convexity, ellipticity.

The first author was partially supported by NSF grant DMS-0905838. The second author was partially supported by RFBR grant 11-01-00302-a. 
the Euclidean unit ball in $\mathbb{R}^{k}$. The resulting area density $A^{b h}: G(n, k) \rightarrow \mathbb{R}_{+}$has the form

$$
A^{b h}(P)=\frac{\varepsilon_{k}}{m_{k}(P \cap B)}
$$

where $B \subset \mathbb{R}^{n}$ is the unit ball of the norm $\|\cdot\|$ and $m_{k}$ is the $k$-dimensional Euclidean area. The corresponding surface area functional has a clear geometric meaning: for embedded surfaces, it is just the $k$-dimensional Hausdorff measure of the surface as a subset of the normed space.

Remark 1.1. Another commonly used notion is the Holmes-Thompson area whose density $A^{h t}$ is given by

$$
A^{h t}(P)=\frac{1}{\varepsilon_{k}} m_{k}\left((P \cap B)^{*}\right),
$$

where $(P \cap B)^{*}$ is the polar body to $P \cap B$ with respect to the Euclidean structure in $P$. Note that $(P \cap B)^{*}$ is the orthogonal projection of $B^{*}$ to $P$ where $B^{*}$ is polar to $B$ in $\mathbb{R}^{n}$.

These two definitions of surface area come from Finsler geometry. A smooth immersed surface in a normed space naturally acquires the induced structure of a Finsler manifold exactly the same way as a surface in Euclidean space gets a Riemannian structure. The surface areas in question can be regarded as Finsler volumes in the induced Finsler metric and belong to its intrinsic geometry.

In Riemannian geometry, Riemannian volume bears two main meanings. Geometrically, it is the Hausdorff measure. Dynamically, it is the projection of the Liouville measure from the unit tangent bundle. In Finsler geometry, there is no notion of volume which would enjoy the two properties. The Busemann-Hausdorff and Holmes-Thompson definitions inherit one of the properties of Riemannian volume each. The Busemann-Hausdorff volume of a $k$-dimensional Finsler manifold $M$ equals the $k$-dimensional Hausdorff measure of the Finsler metric (see [4]). The Holmes-Thompson volume of $M$ (see [10]) is the (normalised by a suitable constant) symplectic volume of the bundle of the unit balls in $T^{*} M$.

The two Finsler volumes can be expressed by the following coordinate formulas (for $\Omega \subset M$ identified with a subset in $\mathbb{R}^{k}$, that is in one chart). For the BusemannHausdorff volume one gets

$$
\operatorname{vol}^{b h}(\Omega)=\varepsilon_{k} \int_{\Omega} m_{k}\left(B_{x}\right)^{-1} d m(x),
$$

and the Holmes-Thompson volume is given by

$$
\operatorname{vol}^{h t}(\Omega)=\frac{1}{\varepsilon_{k}} \int_{\Omega} m\left(B_{x}^{*}\right) d m(x),
$$

where $m_{k}$ is the coordinate Lebesgue measure, $B_{x}$ is the unit ball of the Finsler norm at $x: B_{x}=\left\{v \in T_{x} M: \Phi(v) \leq 1\right\}$. Here we identify $T_{x} M, T_{x}^{*} M$ and $\mathbb{R}^{k}$ since $M=\mathbb{R}^{k}$. The normalizing coefficient $\varepsilon_{k}$ makes the volume definitions agree with the Riemannian one for Riemannian manifolds.

In the above definition of area densities as functions on $G(n, k)$, the Euclidean structure of $\mathbb{R}^{n}$ is irrelevant. Here is an affine-invariant definition. Let $V$ be an $n$-dimensional vector space, $\Lambda^{k} V$ the $k$ th exterior power of $V$, and $G C_{k}(V) \subset$ $\Lambda^{k} V$ the $k$-dimensional Grassmannian cone, that is the set of all simple $k$-vectors. (A $k$-vector $\sigma \in \Lambda^{k} V$ is called simple if it is decomposable: $\sigma=v_{1} \wedge \cdots \wedge v_{k}$ 
for some $v_{1}, \ldots, v_{k} \in V$.) A (translation invariant) $k$-dimensional density in $V$ is a continuous function $A: G C_{k}(V) \rightarrow \mathbb{R}_{+}$which is symmetric and positively homogeneous, that is $A(\lambda \sigma)=|\lambda| A(\sigma)$ for all $\lambda \in \mathbb{R}, \sigma \in G C_{k}(V)$. For a Lipschitz surface $S: M \rightarrow V$ (parametrized by a smooth $k$-dimensional manifold $M$ ), the integral $\int_{S} A$ is defined in an obvious way. We refer to this integral as the $k$ dimensional surface area associated with $A$ and denote it by $\operatorname{Area}_{A}(S)$.

In the case of the Busemann-Hausdorff area in a normed space $(V,\|\cdot\|)$, the density $A^{b h}: G C_{k}(V) \rightarrow \mathbb{R}_{+}$is defined as follows. For a simple $k$-vector $\sigma=$ $v_{1} \wedge \cdots \wedge v_{k}$, the value $A^{b h}(\sigma)$ equals the $k$-dimensional Hausdorff measure (with respect to the metric defined by $\|\cdot\|)$ of the parallelotope spanned by the vectors $v_{1}, \ldots, v_{k}$. It can be expressed by the formula

$$
A^{b h}\left(v_{1} \wedge \cdots \wedge v_{k}\right)=\frac{\varepsilon_{k}}{m_{k}\left(L^{-1}(B)\right)}
$$

where $B$ is the unit ball of $\|\cdot\|$ and $L: \mathbb{R}^{k} \rightarrow V$ is a linear map given by $L\left(e_{i}\right)=v_{i}$ for the standard basis $\left(e_{1}, \ldots, e_{k}\right)$ of $\mathbb{R}^{k}$. We abuse notation and write $A^{b h}(S)$ instead of $\operatorname{Area}_{A^{b h}}(S)$ for the Busemann-Hausdorff area of a surface $S$.

For a Lipschitz chain $S=\sum a_{i} S_{i}, S_{i}: \Delta \rightarrow \mathbb{R}^{n}$, where each $\Delta$ is a standard simplex, we define $\operatorname{Area}_{A}(S)=\sum\left|a_{i}\right| \operatorname{Area}_{A}\left(S_{i}\right)$. The coefficients $a_{i}$ can be taken from $\mathbb{Z}, \mathbb{R}$, or $\mathbb{Z}_{2}:=\mathbb{Z} / 2 \mathbb{Z}$. In the case of $\mathbb{Z}_{2}$, the absolute values $\left|a_{i}\right|$ are defined as follows: $\left|a_{i}\right|=0$ if $a_{i}=0$ and $\left|a_{i}\right|=1$ otherwise. Note that a two-dimensional chain over $\mathbb{Z}$ or $\mathbb{Z}_{2}$ can be parameterized by a manifold (which is oriented in the case of $\mathbb{Z})$.

A density $A: G C_{k}(V) \rightarrow \mathbb{R}$ is said to be convex if it can be extended to a convex function on the vector space $\Lambda^{k} V$ of all $k$-vectors. The functional $A r e a_{A}$ is said to be semi-elliptic over $\mathbb{R}, \mathbb{Z}$, or $\mathbb{Z}_{2}$ (see [1]) if, whenever the boundary $\partial S$ of a chain $S$ over the respective ring is equal to the boundary of a $k$-disc $D$ embedded into an affine $k$-plane, one has $\operatorname{Area}_{A}(S) \geq \operatorname{Area}_{A}(D)$.

It is rather obvious that convexity of $A$ implies semi-ellipticity of $A r e a_{A}$ over $\mathbb{R}$ and $\mathbb{Z}$. The converse is true over $\mathbb{R}$ but in general may fail over $\mathbb{Z}$ (see 3 ).

For the Busemann-Hausdorff surface area density Busemann [5] proved that it is convex in co-dimension one, that is if $\operatorname{dim} V=k+1$, and left the general case as a conjecture (see e.g. [8, p. 37], 6, p. 180] or [11, p. 310, Problem 7.7.1]). The main result of this paper is a proof of this conjecture for the 2-dimensional BusemannHausdorff surface area:

Theorem 1. In every finite-dimensional normed space $V$, the two-dimensional Busemann-Hausdorff area density admits a convex extension to $\Lambda^{2} V$.

Hence the area is semi-elliptic over $\mathbb{Z}$, that is, planar discs minimize the area among orientable surfaces with the same boundary. Furthermore, an easy analysis of the proof shows that it also works in the non-orientable case:

Theorem 2. In every finite-dimensional normed space $V$, the two-dimensional Busemann-Hausdorff area density is semi-elliptic over $\mathbb{Z}_{2}$. That is, every twodimensional affine disc in $V$ minimizes the Busemann-Hausdorff area among all compact Lipschitz surfaces with the same boundary.

Remark 1.2. Since we do not assume that the norm of $V$ is strictly convex, the area functional is not elliptic in general. However it is easy to show that the theorems imply that the Busemann-Hausdorff area is elliptic if the norm is strictly (quadratically) convex. 
Remark 1.3. For the Holmes-Thompson surface area, it had been noticed by Busemann, Ewald and Shephard [7 that the density fails to be convex already for the two-dimensional surface area for a certain norm on $\mathbb{R}^{4}$. Hence it is not elliptic over $\mathbb{R}$ (3), see also 2] for explicit examples.) It turns out however that discs in affine 2-planes minimize the Homes-Thompson area among all surfaces (with the same boundary) parametrized by topological discs (2]). The problem of whether the Holmes-Thomson area is semi-elliptic over $\mathbb{Z}$ (that is, for competing surfaces that may have handles) and beyond dimension 2 remains widely open and intriguing.

The rest of the paper is organized as follows. Sections 2 and 3 are devoted to proofs of Theorems 1 and 2 respectively. The proof of the main Theorem 1 goes via constructing calibrating forms with constant coefficients, and the construction of the forms is based on a certain inequality for the Euclidean area of a convex centrallysymmetric polygon. As it was pointed out by the anonymous referees, the key Proposition 2.2 is connected to investigations related to areas of random triangles carried out by Blaschke and others in response to Sylvester's Four-Point Problem. Namely, the proposition actually describes the probability measure supported on a centrally-symmetric convex curve so as to maximize the expectation of the area of a triangle formed by the center and two random points on the curve.

In Section 4 we discuss prospectives and limitations of our methods for feasible generalizations to higher dimensions.

\section{Proof of Theorem 1}

Definition 2.1. Let $V$ be a finite-dimensional vector space, $A: G C_{k}(V) \rightarrow \mathbb{R}_{+}$a $k$-dimensional density, and $P \subset V$ a $k$-dimensional linear subspace. A calibrator (or a calibrating form) for $P$ with respect to $A$ is an exterior $k$-form $\omega \in \Lambda^{k} V^{*}$ such that for every simple $k$-vector $\sigma \in G C_{k}(V)$, one has $|\omega(\sigma)| \leq A(\sigma)$, and this inequality turns into equality if $\sigma \in \Lambda^{k} P$.

One easily sees that $A$ admits a convex extension to $\Lambda^{k} V$ if and only if every 2-plane $P$ admits a calibrator. (Indeed, an exterior $k$-form is a linear function on $\Lambda^{k} V$, and a calibrator is a linear support for $A$.) Hence our plan is to give an explicit construction of such calibrators for $k=2$ and $A=A^{b h}$.

Let $V$ be a finite-dimensional normed space and $B$ its unit ball. By means of approximation, it suffices to prove Theorem 1 in the case when $B$ is a polyhedron. Fix a two-dimensional linear subspace $P \subset V$. Our goal is to construct a calibrator for $P$ with respect to the Busemann-Hausdorff area density.

Consider the intersection $B \cap P$. It is a centrally symmetric polygon $a_{1} a_{2} \ldots a_{2 n}$ (whose center is the origin 0 of $V$ ). For each $i=1, \ldots, n$, let $F_{i}: V \rightarrow \mathbb{R}$ be a supporting linear function to $B$ such that $F_{i}=1$ on the segment $\left[a_{i} a_{i+1}\right]$. For each $i=1, \ldots, n$, let

$$
p_{i}=\frac{2 A\left(\triangle 0 a_{i} a_{i+1}\right)}{A(B \cap P)}
$$

where $A$ is an (arbitrarily normalized) area form on $P$ and $\triangle 0 a_{i} a_{i+1}$ is the triangle with vertices $0, a_{i}, a_{i+1}$. Note that $\sum p_{i}=1$. Define a 2 -form $\omega \in \Lambda^{2} V^{*}$ by

$$
\omega=\pi \cdot \sum_{1 \leq i<j \leq n} p_{i} p_{j} F_{i} \wedge F_{j} .
$$

We are going to prove that $\omega$ is a desired calibrator for $P$. 
Consider a simple 2-vector $\sigma=v_{1} \wedge v_{2}$ where $v_{1}, v_{2} \in V$ are linearly independent vectors. We need to prove that

$$
\left|\omega\left(v_{1} \wedge v_{2}\right)\right| \leq A^{b h}\left(v_{1} \wedge v_{2}\right)
$$

with equality in the case when $v_{1}, v_{2} \in P$. Identify the plane $\left(v_{1}, v_{2}\right)$ with $\mathbb{R}^{2}$ by means of the linear embedding $I: \mathbb{R}^{2} \rightarrow V$ that takes the standard basis of $\mathbb{R}^{2}$ to $v_{1}$ and $v_{2}$. Then

$$
\left|\omega\left(v_{1} \wedge v_{2}\right)\right|=\left|I^{*} \omega\right|=\pi \cdot\left|\sum_{1 \leq i<j \leq n} p_{i} p_{j} f_{i} \wedge f_{j}\right|
$$

where $f_{i}=I^{*} F_{i}=F_{i} \circ I$ and the norms of 2 -forms are taken with respect to the Euclidean structure of $\mathbb{R}^{2}$. The fact that $F_{i}$ is a supporting function for $B$ implies that $f_{i} \leq 1$ on the set $K=I^{-1}(B)$ which corresponds to the unit ball of the norm restricted to the plane $\left(v_{1}, v_{2}\right)$. By the definition of the Busemann-Hausdorff area,

$$
A^{b h}\left(v_{1} \wedge v_{2}\right)=\frac{\pi}{A(K)}
$$

where $A$ is the Euclidean area. Thus the problem reduces to the following statement from convex geometry on the plane.

Proposition 2.2. Let $K \subset \mathbb{R}^{2}$ be a symmetric convex polygon, $f_{1}, \ldots, f_{n}: \mathbb{R}^{2} \rightarrow \mathbb{R}$ are linear functions such that $\left.f_{i}\right|_{K} \leq 1$ for all $i$, and $p_{1}, \ldots, p_{n}$ are nonnegative real numbers such that $\sum p_{i}=1$. Then

$$
\left|\sum_{1 \leq i<j \leq n} p_{i} p_{j} f_{i} \wedge f_{j}\right| \leq \sum_{1 \leq i<j \leq n} p_{i} p_{j}\left|f_{i} \wedge f_{j}\right| \leq \frac{1}{A(K)} .
$$

In addition, if $K$ is a convex $2 n$-gon $a_{1} a_{2} \ldots a_{2 n}, f_{i}$ are supporting functions of $K$ corresponding to its sides (that is, such that $f_{i}=1$ on $\left[a_{i} a_{i+1}\right]$ ), and $p_{i}=$ $2 A\left(\triangle 0 a_{i} a_{i+1}\right) / A(K)$, then the above inequalities turn into equalities.

The proof of Proposition 2.2 occupies the rest of this section. It consists of several elementary lemmas.

Lemma 2.3. Let $K=a_{1} a_{2} \ldots a_{2 n}$ be a symmetric $2 n$-gon in $\mathbb{R}^{2}$. Let $v_{i}=\overrightarrow{a_{i} a_{i+1}}$ for $i=1, \ldots, n$. Then

$$
A(K)=\sum_{1 \leq i<j \leq n}\left|v_{i} \wedge v_{j}\right|=\left|\sum_{1 \leq i<j \leq n} v_{i} \wedge v_{j}\right|
$$

Proof. The second identity follows from the fact that all pairs $\left(v_{i}, v_{j}\right), 1 \leq i<j \leq n$, are of the same orientation. To prove the first one, observe that

$$
A(K)=2 A\left(a_{1} a_{2} \ldots a_{n+1}\right)=2 \sum_{j=2}^{n} A\left(\triangle a_{1} a_{j} a_{j+1}\right)
$$

since $K$ is symmetric. Further,

$$
A\left(\triangle a_{1} a_{i} a_{i+1}\right)=\frac{1}{2}\left|\overrightarrow{a_{1} a_{j}} \wedge \overrightarrow{a_{j} a_{j+1}}\right|=\frac{1}{2} \sum_{i=1}^{j}\left|v_{i} \wedge v_{j}\right|
$$

since $\overrightarrow{a_{1} a_{j}}=v_{1}+v_{2}+\cdots+v_{j-1}$ and all pairs $\left(v_{i}, v_{j}\right), i<j$, are of the same orientation. Plugging the second identity into the first one yields the result.

The following lemma takes care of the equality case in Proposition 2.2. 
Lemma 2.4. Let $K=a_{1} a_{2} \ldots a_{2 n}$ be a symmetric $2 n$-gon in $\mathbb{R}^{2}$. For each $i=$ $1, \ldots, n$, let $v_{i}=\overrightarrow{a_{i} a_{i+1}}, f_{i}: \mathbb{R}^{2} \rightarrow \mathbb{R}$ the linear function such that $f_{i}=1$ on $\left[a_{i} a_{i+1}\right]$ and $p_{i}=2 A\left(\triangle 0 a_{i} a_{i+1}\right) / A(K)$. Then

$$
p_{i} p_{j}\left|f_{i} \wedge f_{j}\right|=\frac{1}{A(K)^{2}}\left|v_{i} \wedge v_{j}\right|
$$

for all $i, j$, and therefore

$$
\left|\sum_{1 \leq i<j \leq n} p_{i} p_{j} f_{i} \wedge f_{j}\right|=\sum_{1 \leq i<j \leq n} p_{i} p_{j}\left|f_{i} \wedge f_{j}\right|=\frac{1}{A(K)} .
$$

Proof. Denote $S_{i}=2 A\left(\triangle 0 a_{i} a_{i+1}\right)=\left|a_{i} \wedge a_{i+1}\right|$, then $p_{i}=S_{i} / A(K)$. The oriented area form of $\mathbb{R}^{2}$ determines a linear isometry $J:\left(\mathbb{R}^{2}\right)^{*} \rightarrow \mathbb{R}^{2}$ in the standard way, and one easily sees that $J\left(S_{i} f_{i}\right)= \pm v_{i}$ (depending on the orientation). Hence

$$
S_{i} S_{j}\left|f_{i} \wedge f_{j}\right|=\left|\left(S_{i} f_{i}\right) \wedge\left(S_{j} f_{j}\right)\right|=\left|v_{i} \wedge v_{j}\right|
$$

and therefore

$$
p_{i} p_{j}\left|f_{i} \wedge f_{j}\right|=\frac{1}{A(K)^{2}} S_{i} S_{j}\left|f_{i} \wedge f_{j}\right|=\frac{1}{A(K)^{2}}\left|v_{i} \wedge v_{j}\right|
$$

To prove the second assertion, observe that all pairs $\left(f_{i}, f_{j}\right), 1 \leq i<j \leq n$, are of the same orientation, hence

$$
\left|\sum_{1 \leq i<j \leq n} p_{i} p_{j} f_{i} \wedge f_{j}\right|=\sum_{1 \leq i<j \leq n} p_{i} p_{j}\left|f_{i} \wedge f_{j}\right|=\frac{1}{A(K)^{2}} \sum_{1 \leq i<j \leq n}\left|v_{i} \wedge v_{j}\right|=\frac{1}{A(K)}
$$

where the last identity follows from Lemma 2.3 .

It remains to prove the inequality part of Proposition 2.2. The next lemma covers the principal case when the $f_{i}$ 's are supporting functions of the sides.

Lemma 2.5. Let $K=a_{1} a_{2} \ldots a_{2 n}$ be a symmetric $2 n$-gon in $\mathbb{R}^{2}$. For each $i=$ $1, \ldots, n$, let $f_{i}: \mathbb{R}^{2} \rightarrow \mathbb{R}$ be linear function such that $f_{i}=1$ on $\left[a_{i} a_{i+1} \mid\right.$. Let $p_{1}, \ldots, p_{n}$ be nonnegative real numbers such that $\sum p_{i}=1$. Then

$$
\sum_{1 \leq i<j \leq n} p_{i} p_{j}\left|f_{i} \wedge f_{j}\right| \leq \frac{1}{A(K)}
$$

Proof. Denote $v_{i}=\overrightarrow{a_{i} a_{i+1}}, q_{i}=2 A\left(\triangle 0 a_{i} a_{i+1}\right) / A(K)$ and $\lambda_{i}=p_{i} / q_{i}$. By the first part of Lemma 2.5.

$$
q_{i} q_{j}\left|f_{i} \wedge f_{j}\right|=\frac{1}{A(K)^{2}}\left|v_{i} \wedge v_{j}\right|
$$

Let $v_{i}^{\prime}=\lambda_{i} v_{i}$ for $i=1, \ldots, n$. Consider a symmetric $2 n$-gon $K^{\prime}=a_{1}^{\prime} \ldots a_{2 n}^{\prime}$ such that $\overrightarrow{a_{i}^{\prime} a_{i+1}^{\prime}}=v_{i}^{\prime}$. Then

$$
\begin{aligned}
\sum_{1 \leq i<j \leq n} p_{i} p_{j}\left|f_{i} \wedge f_{j}\right| & =\sum_{1 \leq i<j \leq n} \lambda_{i} \lambda_{j} q_{i} q_{j}\left|f_{i} \wedge f_{j}\right| \\
& =\frac{1}{A(K)^{2}} \sum_{1 \leq i<j \leq n} \lambda_{i} \lambda_{j}\left|v_{i} \wedge v_{j}\right| \\
& =\frac{1}{A(K)^{2}} \sum_{1 \leq i<j \leq n}\left|v_{i}^{\prime} \wedge v_{j}^{\prime}\right|=\frac{A\left(K^{\prime}\right)}{A(K)^{2}}
\end{aligned}
$$


where the last identity follows from Lemma 2.3. Therefore

$$
\sum_{1 \leq i<j \leq n} p_{i} p_{j}\left|f_{i} \wedge f_{j}\right|=\frac{A\left(K^{\prime}\right)}{A(K)^{2}} .
$$

Denote $\ell_{i}=\left|v_{i}\right|$ and $\ell_{i}^{\prime}=\left|v_{i}^{\prime}\right|=\lambda_{i}\left|v_{i}\right|$. Let $h_{i}$ denote the distance from the origin to the line containing the side $\left[a_{i} a_{i+1}\right]$. Then $2 A\left(\triangle 0 a_{i} a_{i+1}\right)=h_{i} \ell_{i}$, hence $q_{i}=h_{i} \ell_{i} / A(K)$. Therefore

$$
1=\sum p_{i}=\sum \lambda_{i} q_{i}=\frac{1}{A(K)} \sum \lambda_{i} h_{i} \ell_{i}=\frac{1}{A(K)} \sum h_{i} \ell_{i}^{\prime} .
$$

The last sum is the two-dimensional mixed volume $V\left(K, K^{\prime}\right)$, thus $V\left(K, K^{\prime}\right)=$ $A(K)$. By the Minkowski inequality (which is a special case of the AlexandrovFenchel inequality), we have $V\left(K, K^{\prime}\right)^{2} \geq A(K) A\left(K^{\prime}\right)$. Therefore $A\left(K^{\prime}\right) \leq A(K)$. Substituting this inequality into (2.2) yields the assertion of the lemma.

To complete the proof of Proposition 2.2 it remains to prove the inequality (2.1) in a slightly more general setting, namely when $K$ is a symmetric polygon (not necessarily with $2 n$ sides) and $f_{1}, \ldots, f_{n}$ are arbitrary linear functions such that $\left.f_{i}\right|_{K} \leq 1$. The condition $\left.f_{i}\right|_{K} \leq 1$ means that $f_{i}$ belongs to the polar polygon $K^{*} \subset\left(\mathbb{R}^{2}\right)^{*}$. Consider the left-hand side of (2.1) as a function in one variable $f_{i}$ with others staying fixed. This function is convex (since it is a sum of the absolute values of linear functions), therefore it attains its maximum on $K^{*}$ at a vertex of $K^{*}$. The vertices of $K^{*}$ are supporting linear functions to $K$ at its sides. So it suffices to consider the case when each $f_{i}$ equals 1 on one of the sides of $K$.

If two of the functions $f_{i}$ and $f_{j}$ coincide (without loss of generality, $f_{1}=f_{n}$ ), one reduces the problem to a smaller number of functions as follows: drop $f_{n}$ from the list of functions and replace $p_{1}, p_{2}, \ldots, p_{n}$ by $p_{1}+p_{n}, p_{2}, \ldots, p_{n-1}$. Also note that changing sign of one of the functions $f_{i}$ does not change the left-hand side of (2.1). Thus it suffices to consider the case when $\pm f_{1}, \ldots, \pm f_{n}$ are all distinct. If $n=1$, the left-hand side of (2.1) is zero so the inequality is trivial. If $n>1$, applying Lemma 2.5 to the polygon $K^{\prime}=\bigcap_{i=1}^{n}\left\{x:\left|f_{i}(x)\right| \leq 1\right\}$ yields that

$$
\sum_{1 \leq i<j \leq n} p_{i} p_{j}\left|f_{i} \wedge f_{j}\right| \leq \frac{1}{A\left(K^{\prime}\right)} \leq \frac{1}{A(K)}
$$

since $K \subset K^{\prime}$. This completes the proof of Proposition 2.2 and hence the proof of Theorem 1

\section{Proof of Theorem 2}

Let $V$ be a finite-dimensional normed space and $B$ its unit ball. Let $M$ be a compact two-dimensional smooth manifold with $\partial M \simeq S^{1}$ and $S: M \rightarrow V$ a Lipschitz map such that $\left.S\right|_{\partial M}$ parametrizes the boundary of a 2-disc $D$ lying in a two-dimensional linear subspace $P \subset V$. Our goal is to prove that $A^{b h}(S) \geq$ $A^{b h}(D)$. By means of approximation, we may assume that $B$ is a polyhedron.

The intersection $B \cap P$ is a symmetric polygon $a_{1} a_{2} \ldots a_{2 n}$. Define linear functions $F_{i}: V \rightarrow \mathbb{R}$ and coefficients $p_{i}(i=1, \ldots, n)$ as in the proof of Theorem 1 . Define a function $\alpha: G C_{2}(V) \rightarrow \mathbb{R}_{+}$by

$$
\alpha(\sigma)=\pi \cdot \sum_{1 \leq i<j \leq n} p_{i} p_{j}\left|\left(F_{i} \wedge F_{j}\right) \cdot \sigma\right|
$$


for all $\sigma \in G C_{2}(V)$. Similarly to the proof of Theorem 11. Proposition 2.2 implies that

$$
\alpha(\sigma) \leq A^{b h}(\sigma)
$$

for all $\sigma \in G C_{2}(V)$, and this inequality turns into equality if $\sigma \in \Lambda^{2}(P)$. Indeed, let $\sigma=v_{1} \wedge v_{2}$ where $v_{1}, v_{2} \in V$ are linearly independent vectors and consider the linear embedding $I: \mathbb{R}^{2} \rightarrow V$ that takes the standard basis of $\mathbb{R}^{2}$ to $v_{1}$ and $v_{2}$. Let $K=I^{-1}(B)$, then

$$
A^{b h}(\sigma)=\frac{\pi}{A(K)}
$$

where $A$ is the Euclidean area. On the other hand

$$
\alpha(\sigma)=\pi \cdot \sum_{1 \leq i<j \leq n} p_{i} p_{j}\left|f_{i} \wedge f_{j}\right|
$$

where $f_{i}=F_{i} \circ I$. Recall that $\left.F_{i}\right|_{B} \leq 1$, hence $\left.f_{i}\right|_{K} \leq 1$ for all $i$. By Proposition 2.2, we have

$$
\sum_{1 \leq i<j \leq n} p_{i} p_{j}\left|f_{i} \wedge f_{j}\right| \leq \frac{1}{A(K)}
$$

and (3.11) follows. In the case when $v_{1}, v_{2} \in P$, the equality case of Proposition 2.2 and the construction of $f_{i}$ and $p_{i}$ yields the equality in (3.1).

It remains to show that (3.1) implies the inequality $A^{b h}(S) \geq A^{b h}(D)$. For each pair $i, j, 1 \leq i<j \leq n$, define a linear map $F_{i j}: V \rightarrow \mathbb{R}^{2}$ by

$$
F_{i j}(v)=\left(F_{i}(v), F_{j}(v)\right) \in \mathbb{R}^{2}, \quad v \in V,
$$

and consider the map $F_{i j} \circ S: M \rightarrow \mathbb{R}^{2}$. The Euclidean area $A\left(F_{i j} \circ S\right)$ of this map is given by

$$
A\left(F_{i j} \circ S\right)=\int_{S}\left|F_{i} \wedge F_{j}\right| .
$$

(The term $\left|F_{i} \wedge F_{j}\right|$ here is a two-dimensional density in $V$ given by $\sigma \mapsto\left|\left(F_{i} \wedge F_{j}\right) \cdot \sigma\right|$, $\sigma \in G C_{2}(V)$. Recall that the integration of a density over a surface, orientable or not, is well-defined.) Therefore

$$
\int_{S} \alpha=\int_{S} \sum_{1 \leq i<j \leq n} p_{i} p_{j}\left|F_{i} \wedge F_{j}\right|=\sum_{1 \leq i<j \leq n} p_{i} p_{j} A\left(F_{i j} \circ S\right) .
$$

Similarly, for the planar disc $D \subset P$ we have

$$
\int_{D} \alpha=\int_{D} \sum_{1 \leq i<j \leq n} p_{i} p_{j}\left|F_{i} \wedge F_{j}\right|=\sum_{1 \leq i<j \leq n} p_{i} p_{j} A\left(F_{i j}(D)\right) .
$$

Observe that $F_{i j}(D) \subset F_{i j} \circ S(M)$ because $\left.S\right|_{\partial M}$ is a degree 1 map from $\partial M$ to $\partial D$. Therefore $A\left(F_{i j} \circ S\right) \geq A\left(F_{i j}(D)\right)$ for all $i, j$, and hence the above identities imply that $\int_{S} \alpha \geq \int_{D} \alpha$. By (3.1), we have $A^{b h}(S)=\int_{S} A^{b h} \geq \int_{S} \alpha$, and $A^{b h}(D)=$ $\int_{D} A^{b h} \geq \int_{D} \alpha$ by the equality case of (3.1). Thus

$$
A^{b h}(S) \geq \int_{S} \alpha \geq \int_{D} \alpha=A^{b h}(D)
$$

and Theorem 2 follows. 


\section{REMARKS ON THE HIGHER-DIMENSIONAL CASE}

Although we cannot generalize Theorem 1 to surfaces of dimension $k>2$ at the moment, some of the arguments from Section 2 apply in this case as well. Moreover, the convexity of a $k$-dimensional Busemann-Hausdorff surface area density is equivalent to a $k$-dimensional analogue of Proposition 2.2. In hope that this approach will be useful, we formulate this equivalence as the following proposition.

Proposition 4.1. For every positive integer $k$, the following two assertions are equivalent.

(i) In every finite-dimensional normed space $V$, the $k$-dimensional BusemannHausdorff area density admits a convex extension to $\Lambda^{k} V$.

(ii) For every $n \geq k$ and every central symmetric convex polyhedron $K \subset \mathbb{R}^{k}$ with $n$ pairs of opposite faces $F_{1}, F_{1}^{\prime}, \ldots, F_{n}, F_{n}^{\prime}$ there exist a collection $\mu_{i_{1} i_{2} \ldots i_{k}}, 1 \leq i_{1}<$ $\cdots<i_{k} \leq n$, of real coefficients such that the following holds. For every convex polyhedron $K^{\prime} \in \mathbb{R}^{k}$ and every collection of linear functions $f_{1}, \ldots, f_{n}: \mathbb{R}^{k} \rightarrow \mathbb{R}$ such that $\left.f_{i}\right|_{K^{\prime}} \leq 1$ for all $i$, one has

$$
\left|\sum \mu_{i_{1} i_{2} \ldots i_{k}} f_{i_{1}} \wedge \cdots \wedge f_{i_{k}}\right| \leq \frac{1}{\operatorname{vol}\left(K^{\prime}\right)}
$$

and this inequality turns into equality if $K^{\prime}=K$ and $f_{i}$ 's are supporting linear functions corresponding to faces $F_{i}$ 's (i.e., $\left.f_{i}\right|_{F_{i}}=1$ ).

Remark 4.2. In the two-dimensional case, we just defined $\mu_{i j}=p_{i} p_{j}$ where $p_{i}$ is the portion of the area of $K$ spanned by the $i$ th pair of its faces. This construction is not unique: for many polygons $K$, other choice of $\mu_{i j}$ works as well. In higher dimensions, we have no idea how a suitable collection of coefficients (depending on a polyhedron) could be defined, and the most straightforward generalization of the two-dimensional construction does not work.

Proof of Proposition 4.1. The implication (ii) $\Rightarrow(\mathrm{i})$ is similar to the deduction of Theorem 1 from Proposition 2.2 in Section 2. To see that (i) implies (ii), consider a polyhedron $K$ as in (ii) and equip $\mathbb{R}^{k}$ with a norm $\|\cdot\|$ whose unit ball is $K$. Let $f_{1}^{K}, \ldots, f_{n}^{K}: \mathbb{R}^{k} \rightarrow \mathbb{R}$ be the linear functions corresponding to the faces of $K$, then

$$
K=\left\{x \in \mathbb{R}^{k}:\left|f_{i}^{K}(x)\right| \leq 1, i=1, \ldots, n\right\} .
$$

Hence the linear map $f^{K}: \mathbb{R}^{k} \rightarrow \mathbb{R}^{n}$ given by

$$
f^{K}(x)=\left(f_{1}^{K}(x), \ldots, f_{n}^{K}(x)\right), \quad x \in \mathbb{R}^{k},
$$

is an isometric embedding of the normed space $\left(\mathbb{R}^{k},\|\cdot\|\right)$ into $\mathbb{R}_{\infty}^{n}=\left(\mathbb{R}^{n},\|\cdot\|_{\infty}\right)$ where the norm $\|\cdot\|_{\infty}$ on $\mathbb{R}^{n}$ is defined by

$$
\|x\|_{\infty}=\max _{1 \leq i \leq n}\left|x_{i}\right| \text {. }
$$

Assuming (i), the $k$-dimensional Busemann-Hausdorff area density in $\mathbb{R}_{\infty}^{n}$ admits a convex extension, and therefore there exists a calibrating form $\omega \in \Lambda^{k}\left(\mathbb{R}^{n}\right)^{*}$ for the linear subspace $f^{K}\left(\mathbb{R}^{k}\right)$. The fact that $\omega$ is a calibrator means that for every linear map $f: \mathbb{R}^{k} \rightarrow \mathbb{R}^{n}$ one has

$$
\left|f^{*} \omega\right| \leq \varepsilon_{k} \operatorname{vol}\left(f^{-1}(B)\right)^{-1}
$$

with the equality for $f=f^{K}$, where $\varepsilon_{k}$ is the volume of the Euclidean unit ball in $\mathbb{R}^{k}$ and $B=[-1,1]^{n}$ is the unit ball of $\mathbb{R}_{\infty}^{n}$. Let $\mu_{i_{1} i_{2} \ldots i_{k}}, 1 \leq i_{1}<\cdots<i_{k} \leq n$, be 
the coefficients of $\varepsilon_{k}^{-1} \omega$, that is,

$$
\omega=\varepsilon_{k} \sum \mu_{i_{1} i_{2} \ldots i_{k}} d x_{i_{1}} \wedge d x_{i_{2}} \wedge \cdots \wedge d x_{i_{k}} .
$$

Given a polyhedron $K^{\prime} \subset \mathbb{R}^{k}$ and linear functions $f_{i}$ as in (ii), consider $f=$ $\left(f_{1}, \ldots, f_{n}\right): \mathbb{R}^{k} \rightarrow \mathbb{R}^{n}$. Then

$$
f^{*} \omega=\varepsilon_{k} \sum \mu_{i_{1} i_{2} \ldots i_{k}} f_{i_{1}} \wedge \cdots \wedge f_{i_{k}}
$$

and $\operatorname{vol}\left(f^{-1}(B)\right)^{-1} \leq \operatorname{vol}\left(K^{\prime}\right)^{-1}$ since

$$
f^{-1}(B)=\left\{x \in \mathbb{R}^{k}:\left|f_{i}(x)\right| \leq 1, i=1, \ldots, n\right\} \supset K^{\prime}
$$

Thus (4.2) implies (4.1), and the equality in (4.2) for $f=f^{K}$ yields the equality in (4.1) for $K^{\prime}=K$ and $f_{i}=f_{i}^{K}$.

\section{REFERENCES}

[1] F. J. Almgren Jr, Existence and regularity almost everywhere of solutions to elliptic variational problems among surfaces of varying topological type and singularity structure, Ann. of Math. (2) 87 (1968), 321-391.

[2] D. Burago, S. Ivanov, On asymptotic volume of Finsler tori, minimal surfaces in normed spaces, and symplectic filling volume, Ann. of Math. (2) 156 (2002), no. 3, 891-914.

[3] D. Burago, S. Ivanov, Gaussian images of surfaces and ellipticity of surface area functionals, Geom. Funct. Anal. 14 (2004), no. 3, 469-490.

[4] H. Busemann, Intrinsic area, Ann. of Math. (2) 48 (1947), 234-267.

[5] H. Busemann, A theorem on convex bodies of the Brunn-Minkowski type, Proc. Nat. Acad. Sci. U.S.A. 35 (1949), 27-31.

[6] H. Busemann, Geometries in which the planes minimize area, Ann. Mat. Pura Appl. (4) 55 (1961), 171-189.

[7] H. Busemann, G. Ewald, G. C. Shephard, Convex bodies and convexity on Grassmann cones. I-IV, Math. Ann. 151 (1963), 1-41.

[8] H. Busemann, E. G. Straus, Area and normality, Pacific J. Math. 10 (1960), 35-72.

[9] M. Gromov, Filling Riemannian manifolds, J. Differential Geom. 18 (1983), 1-147.

[10] R. D. Holmes, A. C. Thompson, $n$-dimensional area and content in Minkowski spaces, Pacific J. Math. 85 (1979), 77-110.

[11] A. C. Thompson, Minkowski geometry, Encyclopedia of Mathematics and its Applications, vol. 63, Cambridge University Press, Cambridge, 1996.

Dmitri Burago: Pennsylvania State University, Department of Mathematics, UniverSITY PARK, PA 16802, USA

E-mail address: burago@math.psu.edu

Sergei Ivanov: St.Petersburg Department of Steklov Mathematical Institute, Fontanka 27, St.Petersburg 191023, Russia

E-mail address: svivanov@pdmi.ras.ru 